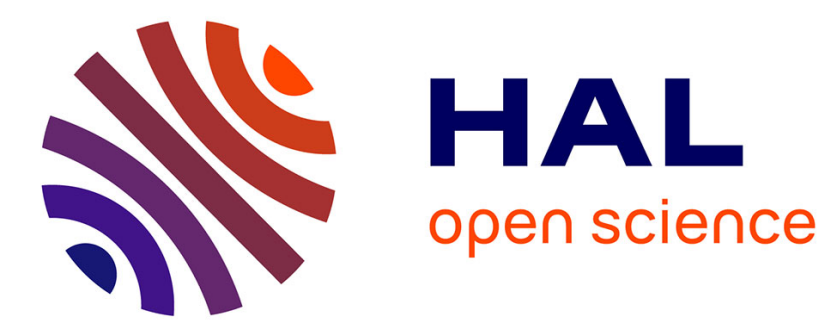

\title{
Les nouvelles configurations migratoires entre l'Europe et les pays du Sud
}

Gildas Simon

\section{To cite this version:}

Gildas Simon. Les nouvelles configurations migratoires entre l'Europe et les pays du Sud. Géographes associés, 2005, 29, pp.59-65. halshs-00688319

\section{HAL Id: halshs-00688319 \\ https://shs.hal.science/halshs-00688319}

Submitted on 17 Apr 2012

HAL is a multi-disciplinary open access archive for the deposit and dissemination of scientific research documents, whether they are published or not. The documents may come from teaching and research institutions in France or abroad, or from public or private research centers.
L'archive ouverte pluridisciplinaire HAL, est destinée au dépôt et à la diffusion de documents scientifiques de niveau recherche, publiés ou non, émanant des établissements d'enseignement et de recherche français ou étrangers, des laboratoires publics ou privés. 


\title{
Les nouvelles configurations migratoires entre l'Europe et les pays du Sud
}

\author{
Gildas SIMON
}

Que se passe-il actuellement sur le plan migratoire du côté du sud de l'Europe ? Je propose de restreindre le champ d'analyse à ce seul versant pour deux raisons: le sujet est à lui seul suffisamment vaste et atteste également d'une opposition classique entre les frontières situées à l'est et à l'ouest de l'Union Européenne. Par ailleurs, d'autres contributions abordent les spécificités des migrations internationales de l'Europe médiane.

Depuis une trentaine d'années, les changements des systèmes migratoires entre l'Union Européenne et les pays situés au sud sont considérables. Par quel mot les nommer, celui d'évolution, de transformation, de recomposition, de chamboulement, de révolution migratoire ? De quelles manières se sont-ils produits, en douceur ou avec des fractures ? Pour le moins, nous sommes confrontés à une véritable mutation profonde et durable.

Ma démarche consistera à retracer ces évolutions en trois temps. Dans une première partie, je proposerai un bref rappel du paysage migratoire pour ce versant sud au début des années 1970. La deuxième partie s'intéressera au rôle de la construction européenne dans la mutation, le changement migratoire des années 1970-2000, et la dernière émettra des hypothèses sur un possible système migratoire unique, en voie de militarisation sur cette façade sud de l'Union Européenne.

\section{Un paysage migratoire bipolaire en forte expansion, mais selon un schéma relativement simple}

Quel est l'état des lieux au début des années 1970 ? De façon très synthétique, quatre caractéristiques principales apparaissent :

- Tous les pays situés au sud de l'Europe sont encore peu ou prou des pays traditionnels de départ. Citons en premier lieu l'Italie, puis l'Espagne et le Portugal, mais également la Yougoslavie (le seul pays à régime socialiste à accepter l'émigration de ses ressortissants) et la Grèce. Il est intéressant de noter qu'un bon nombre de ces pays ont jeté les bases de ce qui a été ou sera une diaspora (grecque, italienne, espagnole, portugaise), cette dernière pouvant fonctionner ultérieurement à rebours. 
- La France, la République Fédérale d'Allemagne et, dans une moindre mesure, la Suisse, sont alors les principaux pôles d'appel, d'emploi et d'immigration des Méditerranéens dans la Communauté Économique Européenne de l'époque. Les Maghrébins se dirigent naturellement et presque exclusivement vers la France ; signalons aussi le démarrage de quelques flux vers la Belgique, les Pays-Bas et l'Allemagne Fédérale. Au début des années 1970, on observe 3 courants migratoires distincts provenant du Maghreb : celui d'Algérie, le plus ancien, le plus enraciné (présence pour certains groupes d'une troisième génération de migrants successifs), et quantitativement le plus important ; celui du Maroc, presque aussi ancien, mais encore limité sur le plan quantitatif, et celui de Tunisie, contemporain de l'indépendance tunisienne. Les Turcs, les Grecs, les Yougoslaves et les Italiens forment alors le gros des effectifs migrants en République Fédérale d'Allemagne.

- Entre pays d'origine au Sud et pays d'emploi au Nord, des champs migratoires spécifiques s'établissent de manière stable, permanente. Ces champs migratoires fonctionnent sur la base d'une circulation migratoire, le va-et-vient, fondé sur des relations culturelles stabilisées, même si des évolutions internes importantes se produisent à l'intérieur comme, par exemple, le développement des migrations familiales et l'apparition de générations nouvelles soit nées dans le pays d'accueil soit arrivées très jeunes dans ce pays.

- La double polarisation des champs migratoires vers la France et l'Allemagne Fédérale structure l'espace des mobilités internationales dans le sud de l'Europe.

À mon sens, on ne peut encore parler, pendant cette période, de façade européenne (sur le plan général et migratoire), comme on parlerait de façade maritime. À cette époque, nous ne sommes en présence ni d'ensembles articulés (chaque pays présente une face différente, spécifique), ni d'un système migratoire sud européen (c'est-à-dire un ensemble coordonné de flux et de dynamiques migratoires fonctionnant en interaction). Chaque pays d'accueil fonctionne par couple migratoire. Nul ne prévoit l'évolution des trois décennies suivantes, sauf à dire que les champs migratoires vont perdurer en raison du différentiel entre la rive nord et la rive sud de la Méditerranée et de la solidité des liens créés par la migration. Telle était à tout le moins la conclusion de ma thèse sur le champ migratoire des Tunisiens en France en 1978.

\section{Quel rôle la construction européenne a-t-elle joué dans la mutation profonde du système migratoire au sud de l'Europe dans les décennies suivantes?}

La réponse à cette question n'est pas évidente car plusieurs mutations sont en cours. De nouvelles dynamiques, d'autres logiques apparaissent qui entrent en interactivité avec les processus et les effets de la construction européenne, dans un contexte économique général et migratoire profondément modifié par la crise de 1973-74.

- Les effets du renversement du contexte migratoire général en Europe. 
La crise de 1973-74, la montée et l'extension du chômage, la désindustrialisation et la précarisation de l'emploi, l'inversion des politiques migratoires nationales en RFA, en France, en Belgique, aux Pays-Bas, le gel des accords de main d'œuvre, les restrictions croissantes à l'entrée, tous ces événements surviennent alors que la pression au départ impulsée par les dynamiques migratoires précédentes perdure et n'a même jamais été aussi forte. Cette nouvelle donne explique la recherche d'autres débouchés, la diversification des flux de départ, la propension à la clandestinité progressive des migrants et le développement considérable des migrations familiales, seule voie légale, acceptable et acceptée. De nouvelles logiques territoriales se développent par extension géographique de la migration marocaine en Europe.

- Le renversement, l'inversion rapide des fonctions migratoires traditionnelles des pays de l'Europe du Sud.

Une autre évolution s'est effectuée à un rythme et une ampleur non prévisibles. Il s'agit du renversement des flux migratoires en Europe du Sud qui s'effectue en l'espace d'une demigénération (1965-1985). La fin de l'onde de départ de nouveaux migrants, déjà amorcée en Italie du Sud au début des années 1960, se réalise à la fin des années 1960 en Espagne et au cours des années 1980 au Portugal et en Grèce. Plusieurs raisons expliquent ces mutations de base: le miracle italien, l'ouverture et la libéralisation des économies avec l'appui non négligeable de l'Union Européenne, l'adaptation de l'économie immergée, le retour de la démocratie et plus simplement, la fin très rapide de la transition démographique, de l'effondrement général de la fécondité et de l'augmentation rapide de l'espérance de vie. L'apparition de nouveaux pays d'immigration, sur le flanc sud, est l'une des cartes majeures de la nouvelle situation migratoire en Europe du Sud.

La recherche de débouchés migratoires et les besoins des marchés de l'emploi se conjuguent pour drainer vers ces nouveaux pays d'immigration les migrants de la rive sud et même audelà. Le nombre d'étrangers croit rapidement, atteignant 2 millions en Italie, 2,2 millions en Espagne, 600000 à 1 million en Grèce, 200000 au Portugal, le tout au prix de nombreuses régularisations. Ainsi, des pays traditionnels d'émigration, d'exode, avec une culture migratoire, des diasporas, se muent en pays d'accueil, d'emploi, d'immigration. La mue est laborieuse, difficile et explique le nombre des grandes opérations de régularisation.

- Quels effets de l'élargissement de l'Europe au sud et de la construction européenne ?

Si l'Italie est membre du noyau fondateur du Traité de Rome, l'entrée de la Grèce en 1981 puis celle de l'Espagne et du Portugal en 1990, modifie la situation. Avec l'élargissement et l'accès à la libre circulation, les pays déjà membres craignaient l'activation ou la réactivation des courants migratoires issus de nouveaux pays entrants. Une période transitoire est prévue pour la libre circulation des travailleurs espagnols et portugais en 1995. En réalité, on a assisté plutôt à l'évolution inverse avec la complexification des formes de mobilité comprenant des retours, des projets de développement, de la double résidence et une circulation migratoire (exemple des Portugais), un affaiblissement de la mobilité nationale et une forte progression de l'immigration étrangère.

Le rôle de la construction européenne et de l'élargissement au Sud est important, voire majeur, dans l'évolution des systèmes migratoires. Cette évolution dans les pays européens du Sud se produit pour un ensemble de raisons complexes. Quelques hypothèses peuvent être formulées : les effets de stimulation des économies nationales et régionales par les aides 
européennes, notamment dans les régions traditionnelles de départ (Nord Portugal), freinent la propension au départ par un sentiment de confiance plus élevé dans l'avenir. De même, l'harmonisation par le haut des politiques et des systèmes de protection sociale dans les pays du Sud, contribue à ces changements. L'Europe sociale finit malgré tout par se construire aussi au sud; d'où cette interrogation : pourquoi partir aujourd'hui ?

Par ailleurs, nous observons chez les migrants du sud des " pays tiers », une modification de l'image, de la représentation du ou plus précisément des pays d'emploi. Alors qu'il y avait une relation quasi unique entre pays d'origine et pays d'arrivée, ce qui concrétisait la réalité du couple migratoire, la polarisation des champs migratoires, il y a maintenant un découplage de la relation, une ouverture des horizons migratoires sur l'ensemble européen. La construction européenne s'est installée progressivement dans les représentations migratoires comme une entité, un effet d'agglomération au niveau de l'image migratoire qui a favorisé la diversification, la dispersion des flux et des courants sur l'ensemble de la façade sud de l'Europe. Mais il faut aussi tenir compte des effets spécifiques des politiques migratoires et des dispositifs réglementaires pour comprendre les évolutions en cours.

\section{Vers un système migratoire unique en voie de militarisation sur la façade sud européenne}

Le concept de système migratoire prend tout son sens. Nous sommes en présence d'un système interactif de processus décisionnels, de dispositifs réglementaires, de dynamiques migratoires qui tendent à unifier l'espace migratoire au sud de l'Europe.

- La mise en place de l'espace Schengen et la construction de la frontière extérieure ont des conséquences dans les pays du Sud

La mise en place de l'espace Schengen et son élargissement sont sans doute l'élément clé du bouleversement migratoire au sud. Selon C.-V. Marie ${ }^{1}$ « La convention de Schengen a été le symbole d'une rupture radicale dans le traitement par les Etats de la question des étrangers et dans l'appréhension de la mobilité internationale ». La volonté de plusieurs Etats dont la France et l'Allemagne de coordonner la lutte contre l'immigration clandestine (obligation du visa par exemple) et le traité de Maastricht font de la liberté de circulation, d'installation et de travail, l'un des attributs essentiels de la citoyenneté européenne. La maîtrise des frontières et de l'asile relève du troisième pilier. En 1997, le traité d'Amsterdam intègre " l'acquis Schengen » et prévoit de faire passer l'immigration du troisième au premier pilier à partir de 1999 pendant une période transitoire de 5 ans et sans réserve de l'unanimité. À terme, la Commission envisage un processus de communautarisation relative au séjour, mais à géométrie variable. Le traité de Nice (décembre 2000) prévoit que les Etats membres passent à partir de 2004 à la majorité qualifiée pour les décisions relatives à l'immigration et à l'asile.

Les questions du contrôle des frontières, de la lutte contre l'immigration clandestine dominent, écrasant le souci d'harmonisation des politiques nationales d'immigration et encore plus, les politiques d'intégration, du « vivre ensemble». La définition de frontière extérieure, dite Schengen, par différence avec les frontières intérieures, désigne la séparation, la ligne de contact aérienne, terrestre ou maritime d'un pays de l'Union avec un Etat tiers. Le contrôle de

\footnotetext{
${ }^{1}$ C.-V. Marie in « La Méditerranée dernière frontière avant l'Europe », COM avril-juin 2003.
} 
cette frontière est devenu un élément, un enjeu majeur de la maîtrise des flux en Méditerranée (responsabilité du contrôle physique et · réglementaire à l'entrée, question de la réadmission des clandestins et des demandeurs d'asile refoulés, Convention de Dublin 1990 ... ). On assiste à l'édification d'un véritable "limes" sur le flanc sud.

Une militarisation de la frontière se met en place avec l'édification de murs fortifiés, de barrières aux points d'accès possibles dans l'enclave espagnole de Ceuta, d'établissement de patrouilles maritimes (on envisage la création d'un corps spécial de surveillance des frontières extérieures), avec l'appui économique des programmes européens pour la construction d'infrastructures de surveillance (système intégral de surveillance extérieure par exemple). Le fonctionnement en façade s'installe, se généralise du côté sud.

La même démarche s'applique aux Etats bordiers de la frontière extérieure avec la définition de la politique du glacis migratoire, en référence aux constructions de Vauban (talus incliné en avant d'une fortification). Il s'agit de s'assurer de la collaboration des Etats bordiers auxquels est confiée, déléguée, moyennant compensation, une fonction d'avant-poste de surveillance de la frontière. Le Maroc est l'exemple type aujourd'hui de ce gardien du glacis migratoire du limes (politiques répressives, militarisation des frontières maritimes et terrestres). Le confinement et le refoulement des non Méditerranéens (arrestation en 2002 de 15000 clandestins subsahariens, détention dans des camps dans des conditions exécrables et expulsion vers l'Algérie et les pays africains) a reçu 40 millions d'euros au titre du programme Europ Meda (2000-2004), condition de la politique d'aide au développement.

- La régionalisation et la mondialisation des flux modifient l'échelle des systèmes, complexifient leur fonctionnement, intègrent de nouvelles dynamiques, compliquent la solution des problèmes

La géographie basique des flux, des dynamiques et des systèmes migratoires demeure, mais l'ensemble du système migratoire évolue par un double phénomène de régionalisation et de mondialisation. De nouvelles relations centre-périphérie s'installent sur les marges en Europe, avec l'arrivée de migrants d'origine Est européenne, Ukrainiens au Portugal, Roumains en Espagne, Polonais et Biélorusses en Grèce. Les logiques migratoires à partir des nouvelles périphéries migratoires de l'Union Européenne prennent corps avec des pays relais vers d'autres pays cibles.

On assiste également à une régionalisation autour de la Méditerranée à la suite de la rupture des couples migratoires. Par leurs origines, leurs itinéraires, leurs destinations prévues et imprévues, les flux, se diversifient, se dispersent dans presque toutes les directions sur le flanc sud de l'Europe, par exemple des Marocains, des Algériens, des Tunisiens cherchent à transiter par la Turquie. Des Éthiopiens soudanais tentent leur chance par les pays du Maghreb, des Kurdes, des Irakiens, des Syriens se dirigent vers les côtes de la Méditerranée occidentale. Nous sommes face à un brouillage généralisé des cartes migratoires traditionnelles.

Mais le phénomène sans doute le plus important est l'africanisation du système migratoire méditerranéen. L'irruption de nouveaux acteurs migratoires, de nouvelles dynamiques en provenance de l'Afrique subsaharienne et de l'Afrique du Nord-Est (Soudan, Ethiopie, Erythrée) installe la connexion migratoire, intracontinentale, transsaharienne entre l'Afrique noire subsaharienne et la rive sud de la Méditerranée. Sénégalais, Gambiens, Ghanéens, 
Nigérians, Congolais remontent par des filières organisées vers le Maroc, la Tunisie, la Libye pour tenter la traversée des détroits. Ce transit est réel, dangereux, mortifère mais il est aussi une impasse et implique la dégradation locale de leurs conditions d'existence.

L'Europe est aussi confrontée à l'arrivée et la diffusion de migrants d'Amérique du Sud (fonctionnement à rebours des empires coloniaux et de diasporas de l'Europe méditerranéenne). En 2003, les Équatoriens sont le premier groupe étranger en Espagne, soit 390000 devant les Marocains (380 000) et les Colombiens (240000). D'autres migrants d'Asie du Sud et de l'Est comme les Philippins arrivent en Italie et Espagne, des Chinois sont en Italie.

Ces nouvelles donnes expliquent les difficultés croissantes de ce changement d'échelles, de dialogues entre l'Union Européenne et les pays de départ extérieurs à la zone. Quels sont les interlocuteurs? Quels sont les moyens d'action?

- Nouveaux acteurs, nouveaux enjeux, nouveaux défis : du champ migratoire aux champs transnationaux d'opportunité

L'Europe est confrontée à la venue de nouveaux migrants mais également à de nouvelles pratiques, à des perceptions différentes, à des logiques fondées sur d'autres objectifs que ceux de la migration traditionnelle. Les acteurs traditionnels et les nouveaux venus contribuent à la complexification des réseaux migratoires, des configurations diasporiques, qui se combinent avec la circulation des entrepreneurs, et des trabendistes. Le chamboulement est total. La fin des couples migratoires se combine avec la multipolarisation des champs migratoires.

L'Union Européenne est aussi confrontée à l'accélération du processus de l'élargissement vers le sud avec l'entrée de Malte et surtout de Chypre. Les conséquences de cette position stratégique au coeur de la Méditerranée orientale sont encore peu décelables.

À ce tableau, encore incomplet, il faut aussi ajouter la prévision des enjeux démographiques des migrations méditerranéennes, la pression migratoire intrinsèque dans les pays de l'est et du sud de la Méditerranée (PESM), le vieillissement démographique au Nord, les enjeux socioculturels de l'Islam, le défi du terrorisme, etc ...

Nous sommes confrontés à une nouvelle surenchère dans les dispositifs de surveillance, révélant la montée d'une suspicion généralisée.

\section{Conclusion}

Il est difficile de prévoir les tendances migratoires générales, spécialement en Méditerranée en raison du risque géopolitique qui y est plus présent qu'ailleurs. Mais il est possible d'avoir quelques certitudes. La pression migratoire restera forte pendant plusieurs décennies en raison du poids des jeunes actifs sur le marché du travail, les retards du développement local, les frustrations et les attentes sociales et l'on sait que la demande liée au vieillissement au Nord augmentera. Ainsi, la mondialisation du système migratoire sud Européen se poursuivra. 